\title{
ARE THERE PROMINENCES AROUND BINARY SYSTEMS?
}

\author{
R. D. JEFFRIES \\ Department of Physics, Keele University, Keele, Staffordshire \\ ST5 $5 B G, U K$ \\ School of Physics and Space Research, University of Birmingham, \\ Birmingham B15 2TT, UK
}

\begin{abstract}
I examine the possibility that large, cool prominences can be formed in close binary systems, when coronal structures grow beyond the point where centrifugal force balances gravitational attraction. X-ray light curves of the eclipsing binary XY UMa indicate that an extended corona is probably present and this is likely to reach heights $>1.5 \mathrm{R}_{\odot}$, where centrifugal compression may render plasma in the coronal loop apexes unstable to thermal perturbations. Cool condensations could form, explaining features such as the dips in the X-ray light curves of V471 Tau, and the circumstellar material deduced to be present in several close binary systems.
\end{abstract}

\section{Introduction}

In recent years the evidence has been growing that there are extended regions of cool material in the outer atmospheres and coronae of active stars. It is now well established that co-rotating clouds of neutral material, trapped in giant magnetic loops, provide an attractive explanation for the transient $\mathrm{H} \alpha$ absorption features seen in single, rapidly rotating stars (Collier Cameron \& Robinson 1989; Collier Cameron \& Woods 1992; Jeffries 1993; Collier Cameron - these proceedings). In these stars it is observed that the prominences appear to lie outside the Keplerian corotation radius and are likely to be produced by thermal instabilities inherent to the centrifugally compressed plasma present in magnetically constrained coronal loops of extent several $R_{\odot}$ (Collier Cameron 1988).

It is of interest to ask whether the same kind of structures are present in close binary systems, where rapid rotation could also centrifugally compress extended coronal plasma. Hall \& Ramsey $(1992,1994)$ use high resolution Balmer line spectroscopy to argue that there are substantial amounts

461

K. G. Strassmeier and J. L. Linsky (eds.), Stellar Surface Structure, 461-468.

(c) 1996 IAU. Printed in the Netherlands. 
of cool, prominence-like material in extended structures around many binary systems. Young, Rottler \& Skumanich (1991) reported a similar phenomenon around the eclipsing white-dwarf/K-star binary, V471 Tau. Furthermore, in this system, soft X-ray dips have been observed, which could be interpreted as absorption by neutral material at the L4 and L5 points (Jensen et al. 1986). Jeffries (1991) has shown, using X-ray and UV data, that the discrepant coronal and transition region pressures in AR Lac system cannot be explained in terms of a conventional model where the transition region emission arises solely from the base of coronal loops. Instead, a large volume of low pressure, cool plasma must exist in the system and may contribute the majority of flux in transition region emission lines.

In this paper a further contribution to the debate is presented in the form of ROSAT Position Sensitive Proportional Counter (PSPC) observations of the eclipsing binary XY UMa. An attempt is made to determine the spatial location and extent of the coronal plasma and relate this to the possibility of centrifugally induced thermal instabilities in the constraining magnetic loop structures.

\section{Observations}

$\mathrm{XY} \mathrm{UMa}$ is an excellent target for $\mathrm{X}$-ray eclipse mapping studies, consisting of a slightly oversized G2V primary and K5V secondary, separated by $3.01 \mathrm{R}_{\odot}$, in a detached $0.48 \mathrm{~d}$ orbit. The primary eclipse is annular and the secondary eclipse total. The system parameters have been recalculated by Hilditch \& Bell (1994), who obtained photometry that is almost cotemporal with the X-ray observations presented here. The ROSAT PSPC observations were performed between 6 October and 1 November 1992. The majority of the total $37 \mathrm{ks}$ of observation was concentrated between 27 October and 1 November and covered several orbital cycles with repeated phase coverage. Figure 1 shows a background subtracted 0.1-2.0 keV light curve of the observation, where each observing slot has been allocated a bin. It is immediately obvious that the X-ray brightness of XY UMa varies by up to a factor of 3 , perhaps due to rotational modulation, but more likely due to flaring behaviour. XY UMa is a known X-ray flaring source (Jeffries $\&$ Bedford 1990), and it is for this reason that repeated phase coverage is essential to discern any true rotational modulation of long-lived coronal structures.

Figure 2 shows the data between 27 October and 1 November, phase folded according to the ephemeris of Hilditch \& Bell (1994). The data have been put into bins of approximately $380 \mathrm{~s}$. The reason for this is to largely eliminate the effect of time-varying obscuration by the PSPC wire structure as the spacecraft oscillates with a period of about 6 minutes. Similar time- 


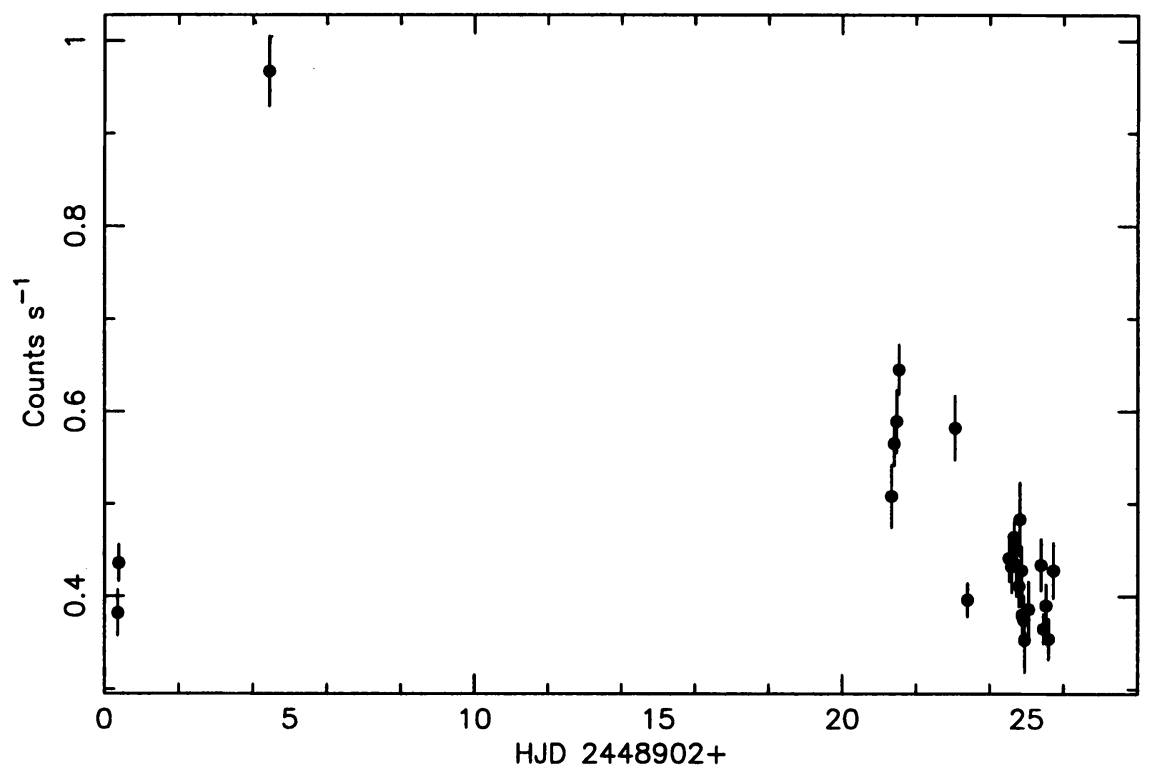

Figure 1. Time series (0.1-2 keV) of XY UMa between 6 October and 1 November 1992.

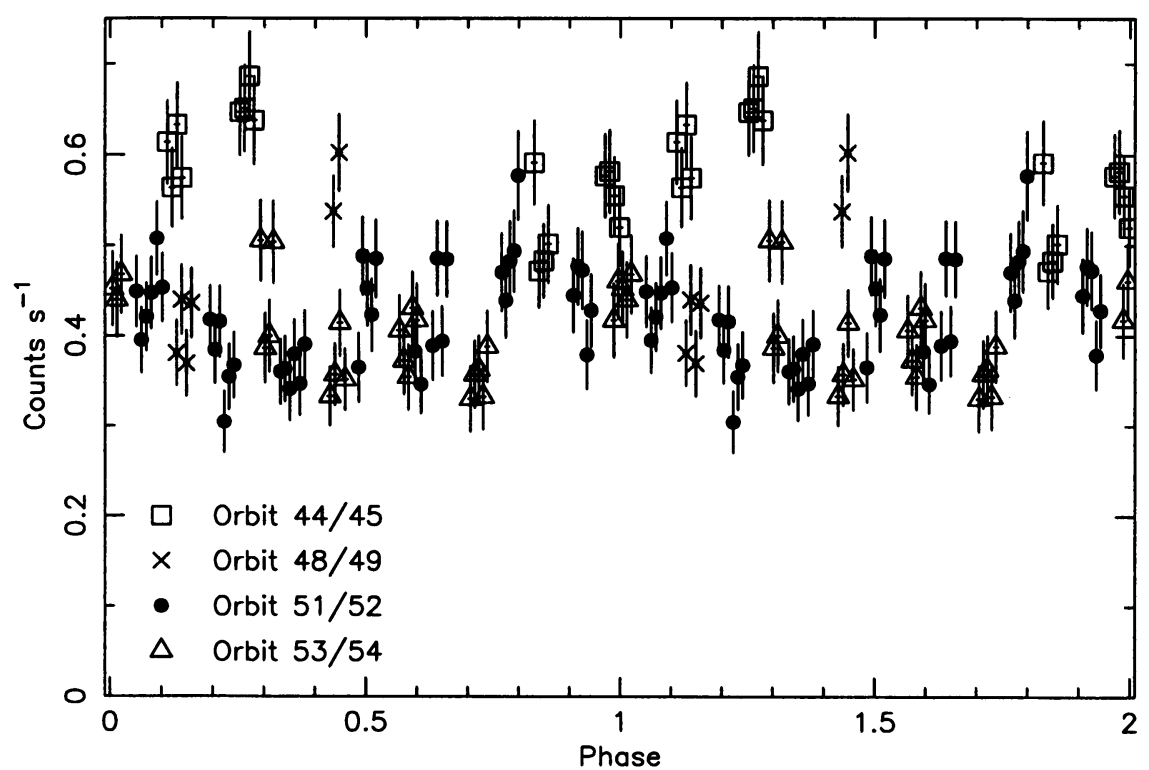

Figure 2. Phase folded time series (0.1-2 keV) of XY UMa between 27 October and 1 November 1992. Orbit numbers refer to binary periods since the start of the ROSAT observation. Phase 1.0 is primary eclipse. 
series were constructed for nominal "hard" $(H=0.5-2.0 \mathrm{keV})$ and "soft" ( $S=0.1-0.4 \mathrm{keV}$ ) bands. Separate, approximately continuous, intervals of data have been allocated different symbols corresponding to the number of binary periods since the start of the ROSAT monitoring. It is clear that there is orbit to orbit variability, even outside of the obvious flare in Figure 1, which occurred in orbit 9 . This makes it very difficult to interpret the data in terms of static coronal structures. The timescale for variability appears to be of order 1 day. No sign of either a primary or secondary eclipse is seen, although it appears that even if the points from orbit $44 / 45$ are excluded there is some evidence for a $10-20$ percent reduction in the flux between phases 0.2 to 0.7 compared to the flux between phases 0.7 to 1.2 .

The phase folded $\mathrm{S}$ band and $\mathrm{H}$ band light curves have a similar appearance to Figure 2. There is no evidence for primary or secondary eclipses and there is no sign that the lower energy flux is significantly more modulated than the higher energy flux, in contrast to some investigations of other binary systems (e.g. AR Lac - White et al. 1990). The hardness ratio (defined as $(H-S) /(H+S))$, shows essentially random variations, with no evidence for periods of time when the visible corona(e) was hotter or cooler, even during the flare in orbit 9.

\section{Modelling}

Spectral modelling with a Raymond \& Smith code was performed, in order to obtain physical parameters for the coronal plasma. No large temporal temperature variations are present in the data, as judged from the hardness ratio. Data from orbits 51 to 54 are selected as representative of a "steady state" light curve, in order to derive the plasma emission measure. An isothermal plasma is excluded by the data at high confidence. Even a two temperature model, with a free absorbing column density, $N_{H}$, is a relatively poor fit, although formally acceptable at the $5 \%$ level $\left(\chi^{2}=23,14\right.$ dof $)$. The best-fit parameters are $T_{\text {low }}=(2.32 \pm 0.04) \times$ $10^{6} \mathrm{~K}, E M_{\text {low }}=(5.2 \pm 0.6) \times 10^{52} \mathrm{~cm}^{-3}$ (assuming a distance of $\left.100 \mathrm{pc}\right)$, $T_{\text {high }}=(1.1 \pm 0.1) \times 10^{7} \mathrm{~K}, E M_{\text {high }}=(1.30 \pm 0.13) \times 10^{53} \mathrm{~cm}^{-3}$ and $N_{H}=(2.4 \pm 1.4) \times 10^{19} \mathrm{~cm}^{-2}$.

To investigate the possible extent of the corona(e), a code has been developed to model particular plasma distributions under a variety of assumptions, and to generate the corresponding X-ray light curves. First, the case of a spherically symmetric, isothermal, isobaric corona around the primary star is considered. Coronal loop models indicate that an isothermal approximation is justified for a given loop because of the efficacy of conductive heat transport, but the isobaric assumption will not in general be 

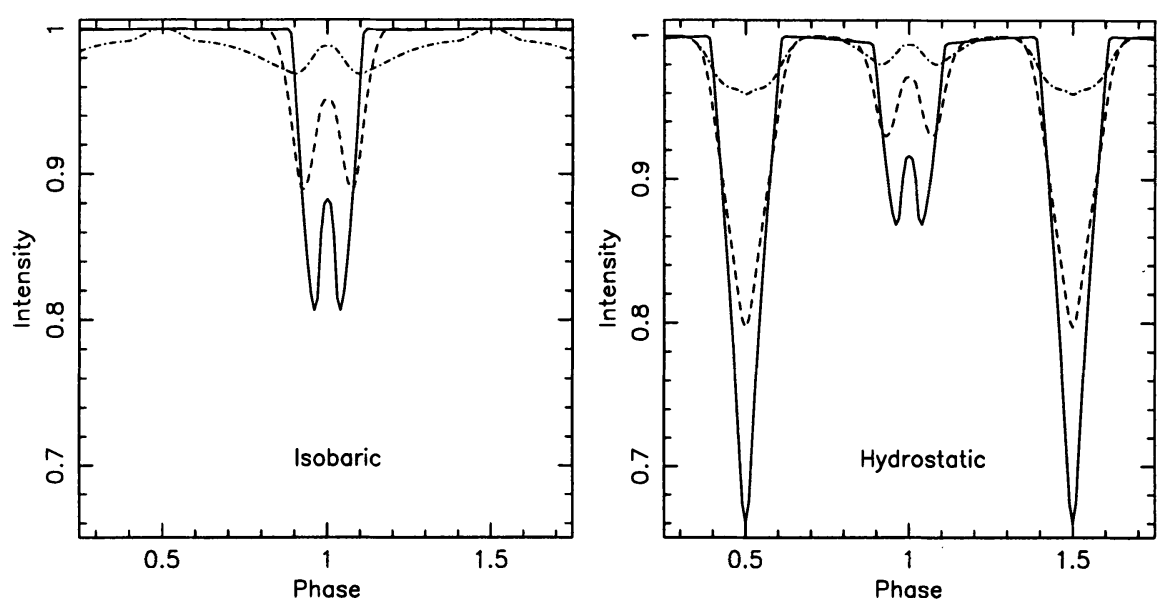

Figure 3. (a) X-ray light curves due to a spherically symmetric, isobaric corona around the primary star of height $0.2 \mathrm{R}_{\odot}$ (solid line), $1.0 \mathrm{R}_{\odot}$ (dashed) and $3.0 \mathrm{R}_{\odot}$ (dash-dot). (b) Light curves due to hydrostatic coronae around both stars with similar heights to (a).

satisfied and will subsequently be removed. Figure 3a shows the X-ray light curve obtained for a number of assumed coronal heights. Notice that the minima are slightly displaced from the centre of primary eclipse, because of the optically thin nature of the plasma. These minimum phases are well observed in orbits $51 / 52$, and there is no evidence for any slope or reduction in the X-ray flux with respect to the rest of the light curve, certainly at the $10 \%$ level. From this, it is estimated that any uniformly distributed corona around the primary star must have a height greater than about $1 R_{\odot}$.

We can now investigate the relaxation of some of the assumptions leading to Figure 3a. A number of factors would lead to greater modulation of the light curve and deeper eclipses, thus implying even larger scale heights for the coronal loops. These are the addition of a corona around the secondary star, axial asymmetry in the plasma distribution, the removal of the isobaric assumption and any concentration of the plasma towards the orbital plane. Figure $3 \mathrm{~b}$ shows light curves generated from coronae with the same extents as Figure 3a, but with plasma anchored to both primary and secondary stars and with hydrostatic atmospheres calculated from the system parameters given in Hilditch \& Bell (1994). A plasma temperature of $10^{7} \mathrm{~K}$ is assumed, and the pressure normalised to the pressure at the surface of the primary star. The net effect of these changes is to make the primary eclipse shallower, but a deeper secondary eclipse is now present. In order to limit the extent of any secondary eclipse, it is again found that plasma extents in excess of $1.5 R_{\odot}$ are required. This restriction is more rigorous 
than in the isobaric case because the emission is concentrated into a $n_{e}^{2}$ scale height around both stars, where $n_{e}$ is the electron density. Note that a trade-off is possible between the depths of the primary and secondary eclipses, by assuming a higher or lower base pressure for the secondary corona. However, it is found that lowering the secondary base pressure sufficiently to bring up the secondary eclipse to a comparable depth with the primary eclipse still results in deeper eclipses than a simple isobaric corona around the primary star. A more trivial way to reduce the depth of any eclipses is to increase the amount of uneclipsed plasma in the polar regions. However, Hilditch \& Bell (1994) find evidence from the almost co-temporal photometry, for significant coverage of equatorial starspots on the primary. These were preferentially distributed on the hemisphere facing the secondary. It seems more likely that any extended plasma will be constrained within large scale dipole field structures, co-aligned with the rotation axis. In which case, plasma is concentrated toward the orbital plane and even greater modulation of the X-ray light curve would be expected. In conclusion, it is suspected that the coronal plasma around XY UMa is constrained in large scale fields with heights of at least $1 R_{\odot}$ and probably considerably more.

\section{Implications}

If the coronal plasma is constrained in a large scale dipole field, then the constraint that the extent $>1 \mathrm{R}_{\odot}$, leads to pressures of 15 dyne $\mathrm{cm}^{-2}$ and coronal field strengths of at least $20 \mathrm{G}$, or several hundred $\mathrm{G}$ at the stellar surface. Of course, if the filling factor is less than 1 , then these numbers become correspondingly larger, but are not inconsistent with the idea that large fractions of the stellar surface are covered by kilogauss fields.

Turning now to the pressure distribution of this plasma, which is illustrated in Figure 4, for slices through the primary star, perpendicular to the line of centres and along the line of centres respectively. The coronal extent has been set at $2.2 \mathrm{R}_{\odot}$ around the primary star only, and the plasma is constrained in a dipole field. One can see immediately that there is a change in direction of the pressure derivative with height above the surface. The height above the stellar surface where this occurs is dependent upon stellar longitude. For the quadrature directions it is about $1.55 \mathrm{R}_{\odot}$, but opposite the sub-stellar point it is only $1.25 \mathrm{R}_{\odot}$. Collier Cameron (1988) has shown that coronal loops which protrude beyond this kind of pressure minimum are unlikely to be thermally stable, because of a combination of centrifugal compression and increasing radiative losses with decreasing temperature. If there are heating perturbations, or the loops are filled explosively by chromospheric evaporation, then the plasma above the pressure minimum 

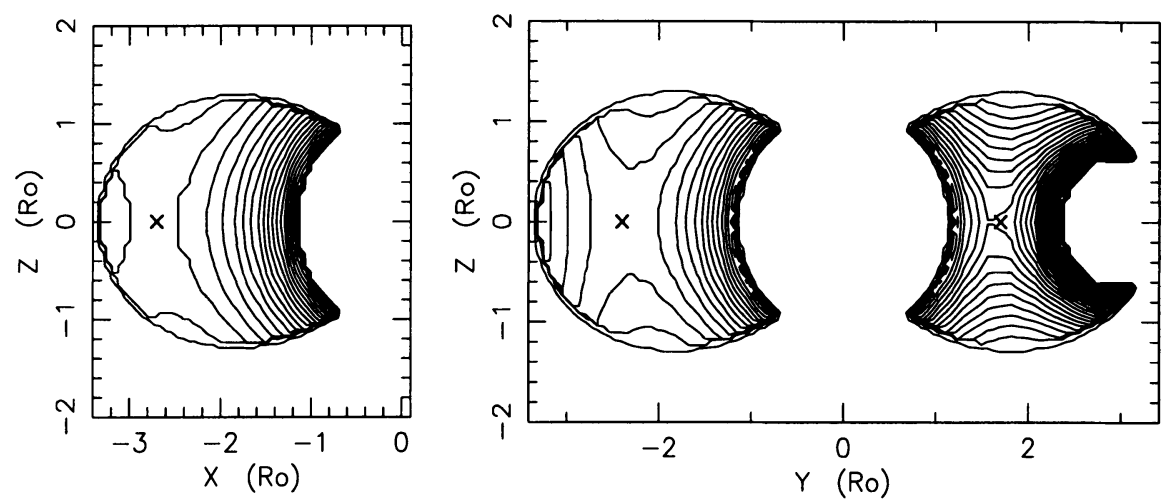

Figure 4. (a) A pressure plot showing a slice through the centre of the primary star, perpendicular to the line of centres (the y-axis). The cross marks the pressure minimum at about $65 \%$ of the surface pressure. Contours are steps of $2 \%$ of the surface pressure. The plot is symmetric about $x=0$. The centre of the primary star is at $(0,0)$. (b) Same as (a) but for a slice along the line of centres. The outer minimum point is at $72 \%$ of the surface pressure, the minimum between the stars at $89 \%$ of the surface pressure.

will be trapped and will cool on timescales governed by radiative and conductive heat losses. For pressures of $\sim 10$ dynes $\mathrm{cm}^{-2}$ and characteristic temperature gradient lengths of $1 R_{\odot}$, these timescales are a few hours to a day, which may go some way to explaining the orbit to orbit variability seen. The situation for plasma between the two stars is less clear. It is possible that this may be held in loops anchored to both stars, in which case there is no site to trap the cooling plasma. Thus the most promising sites for prominence formation in coronal loops anchored to the primary, would be an arc in the orbital plane subtending about $270^{\circ}$, facing away from the secondary. Conversely, for plasma anchored to the secondary, a similar arc facing away from the primary would be the preferred site of prominence formation. So there could be a ring of cooling prominences trapped in coronal loops, around the binary system as a whole.

Although this idea has been discussed with reference to ROSAT observations of XY UMa, there have been several investigations that seem to require extended regions of X-ray emission around other close binary systems such as AR Lac and ER Vul (e.g. White et al. 1990; Culhane et al. 1990). Coronal heights of as much as $3 R_{\odot}$ have been proposed. In both these systems such a corona would have regions lying beyond the point where centrifugal forces balance gravity, so there is similar potential for prominence condensation.

This mechanism provides an attractive explanation for several of the observational points mentioned in the introduction. Cooling prominences may well be responsible for at least some transition region line emission 
at pressures roughly two orders of magnitude lower than the coronal pressure. Eventually the material will become cool enough to provide a source of X-ray absorption, although even a modest amount of ionization will keep the plasma tied to the magnetic field lines. For a coronal pressure of 10 dynes $\mathrm{cm}^{-2}$ and loops extending, say $10^{10} \mathrm{~cm}$ beyond the force balance point one, might expect X-ray absorbing columns of a few $10^{19} \mathrm{~cm}^{-2}$, which is just the sort of figure able to explain the soft X-ray dips seen in V471 Tau by Jensen et al. (1986). The total mass of plasma present depends on the loop geometry. Loop areas of $\sim 10^{21} \mathrm{~cm}^{2}$ would give a prominence mass of about $10^{17} \mathrm{~g}$, approximately two orders of magnitude more massive than solar prominences. As the material becomes largely neutral it will scatter chromospheric $\mathrm{H} \alpha$ photons out of the line of sight and produce absorption transients when seen against the disks of the component stars. I suggest that this is a plausible explanation for the features observed and mapped by Hall \& Ramsey $(1992 ; 1994)$. Doppler mapping of the $\mathrm{H} \alpha$ lines in XY UMa should show similar features. Ultimately the centrifugally compressed plasma will cool sufficiently to diffuse out of the field, or the curvature of the field will increase and perhaps eventually a reconnection event will occur. In either case, it seems probable that the material will escape from the system, carrying angular momentum commensurate with its distance from the rotation axis.

\section{References}

Collier Cameron, A. (1988), MNRAS, 233, 235

Collier Cameron, A. and Robinson, R. D. (1989), MNRAS, 236, 57

Collier Cameron, A. and Woods, J.A. (1992), MNRAS, 258, 360

Culhane, J.L., White, N.E., Shafer, R.A. and Parmar, A.N. (1990), MNRAS, 243, 424

Hall, J.C. and Ramsey, L.W. (1994), AJ, 107, 1149

Hall, J.C. and Ramsey, L.W. (1992), AJ, 104, 1942

Hilditch, R.W. and Bell, S.A. (1994), MNRAS, 267, 1081

Jeffries, R.D. (1991), MNRAS, 253, 369

Jeffries, R.D. (1993), MNRAS, 262, 369

Jeffries, R.D. and Bedford, D.K. (1990), MNRAS, 246, 337

Jensen, K.A., Swank, J.H., Petre, R., Guinan, E.F., Sion, E.M. and Shipman, H.L. (1986), ApJ, 309, L27

White, N.E., Shafer, R.A, Horne, K., Parmar, A.N. and Culhane, J.L. (1990), ApJ, 350, 776

Young, A., Rottler, L. and Skumanich, A. (1991), ApJ, 378, L25 